\title{
NEURAL NETWORKS-BASED MODELS FOR GREENHOUSE CLIMATE CONTROL
}

\author{
Isaías González Pérez, Antonio José Calderón Godoy \\ Escuela de Ingenierías Industriales, Universidad de Extremadura, \{igonzp, ajcalde $\}$ @ unex.es
}

\begin{abstract}
Greenhouses are multivariable and nonlinear systems with high degree of complexity, so it is hard to build models that represent the whole dynamics of the system. This paper presents models of greenhouse climate based on neural networks. The models predict inside air temperature and relative humidity in the greenhouse as a function of the variables used as input for the network, as outside temperature, relative humidity, solar radiation, etc., and the actuators state signals, as window opening and others. Data sets used for modelling have been measured with real red pepper plants inside the greenhouse. The developed models are described and the achieved results are reported.
\end{abstract}

Keywords: neural network, greenhouse climate, control.

\section{INTRODUCTION}

The main goal of greenhouse control is to provide optimal climatic conditions for the growth of inside culture. Greenhouse microclimate is defined by a series of variables which can be regulated to control the development of the hosted crop. In addition, environmental control of greenhouses aims to reduce energy consumption and to raise the efficiency of used resources while maintaining a high crop quality. Figure 1 outlines the control loop scheme of an automated greenhouse.

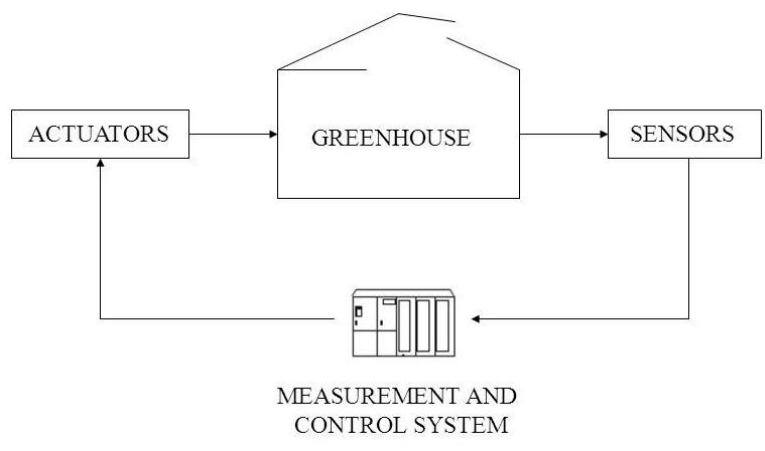

Figure 1: Scheme of automated greenhouse
Greenhouse dynamic behavior depends on the interactions between its main components (internal atmosphere, crop, soil and structure) and on the outside weather fluctuations and on the exerted control actions. Internal microclimate conditions are the result of complex and interactive processes of physical, chemical and biological nature, creating difficulties in controlling the greenhouse climate. Therefore, a greenhouse is a nonlinear, multivariable, non-stationary system and an "open" to exterior environment [1].

Methods aimed at efficiently controlling the greenhouse must take these influences into account, and that is achieved by the use of models [7]. These models describe the system dynamics and are used by the controller to establish the control action to exert. Model-based control techniques need accurate and reliable models of the greenhouse to provide precise and effective climate control results. For these reasons, greenhouse control and modeling problem continues to require important efforts from researchers and engineers in various fields, although it has been studied during the last two decades. Techniques for developing models of real processes can be divided into two main methods. One is based on the physical laws involved in the process [3,4], and the other one is based on the analysis of the process input-output data and from empirical expertise. The first method uses the thermodynamic properties of the greenhouse trough energy and mass flows equations. In general, these models have a high degree of complexity with lots of parameters that have to be determined by calibration. The second method is based on a system identification approach using linear and nonlinear techniques. These models do not allow investigating the internal functioning of the system, but they can be very helpful for climate control purposes.

Artificial Intelligence techniques have been widely applied to obtain greenhouse environment models, such as fuzzy logic [14], and neural networks [6-10, $12,13,17]$. Even, hybrid models, combination of physical models and neural networks, have been also applied to greenhouse modeling [11]. Recently published papers dealing with neural network-based models applied to greenhouses are [5] and [16]. As demonstrated by those works, the method of 
greenhouse identification by means of neural networks provides reliable models which can reflect the nonlinear characteristics of the greenhouse system.

Commercial controllers do not provide flexibility for researching and testing of different control methods since they are closed systems for the final user. Besides, in many cases, a precise manipulation of climate variables is not reachable because the equipment do not adapt to specific requirements of greenhouse in which they are installed. Controllers are designed for greenhouses with different characteristics such as materials, structure and other constructive factors, and also, for geographical locations with different weather conditions.

However, better climate management results can be achieved by means of a control system properly adapted to the greenhouse. These issues are emphasized in Extremadura (south-west Spain), region characterized by dry weather with high temperatures in summer and cold winters, where the greenhouse to be modeled is located.

The aim of this research is to develop neural models of the greenhouse with data retrieved from real red pepper plants cultivation. These models are envisioned to be used for model-based control strategies.

The paper is organized as follows. Section 2 is dedicated to describe the considered greenhouse and the used data sets. Section 3 covers neural networks and the achieved results. Finally, section 4 presents the main conclusions and future works.

\section{MATERIALS AND METHODS}

\subsection{GREENHOUSE DESCRIPTION}

Figure 2 shows the aspect of the greenhouse that has been used in the present work. It is a pitched-roof greenhouse with aluminum armature, glass walls and concrete floor. It is defined by a surface of $36 \mathrm{~m}^{2}$ and $3 \mathrm{~m}$ of average height. Plants are distributed over several cultivation trays. Attached to one wall there is a small header house of polycarbonate sheets that serves as control room. It contains a commercial measurement and control system which automates the greenhouse by means of several actuators and sensors.

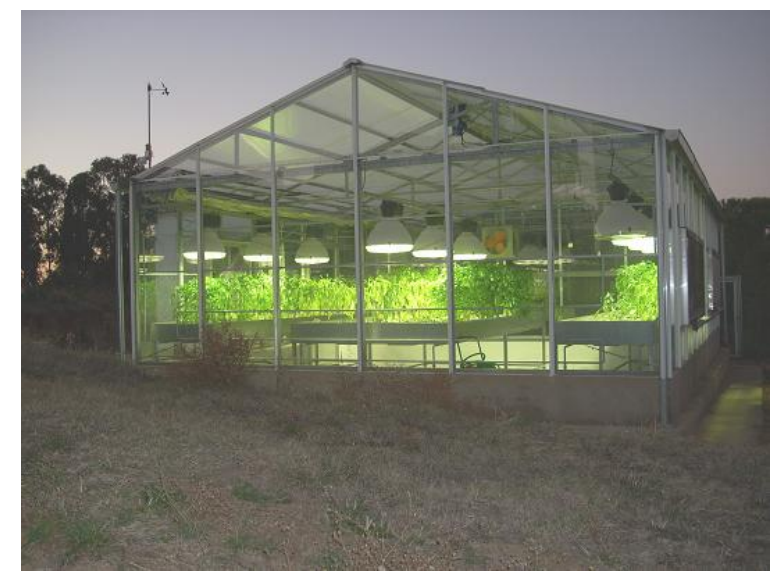

Figure 2: Photograph of the greenhouse

Figure 3 depicts the functional block diagram of the experimental greenhouse. The measured variables can be classified into the groups: weather disturbances, control inputs and control outputs. Inside microclimate is defined by the inside temperature $(\mathrm{Ti})$ and humidity $(\mathrm{Hi})$, hence these variables constitute the greenhouse control outputs. Actuators for climate management which act as control inputs are the followings: a roof window for natural ventilation $(O p)$, a heating system $(C h)$, a thermal/shadow screen $(S c)$, a cooling system based on evaporative pads and a fan $(C s)$, and a set of artificial lighting lamps $(L a)$. Outside weather conditions affect to greenhouse microclimate and constitute important disturbances for the system. The following external variables are measured: external temperature $(\mathrm{Te})$, external humidity $(\mathrm{He})$, wind speed $(V w)$, wind direction $(D w)$ and rain detection $(R d)$. Besides, the global radiation $(R g)$ is acquired by a meteorological station located close to the greenhouse.

Inside air environmental data (temperature and humidity) are measured at the central point of the greenhouse.

The aforementioned measurement and control system implements an On-Off control strategy. The control program evaluates the user setpoints and the constraints in order to determine and adjust the ranges where the regulated environmental parameters must be maintained. 


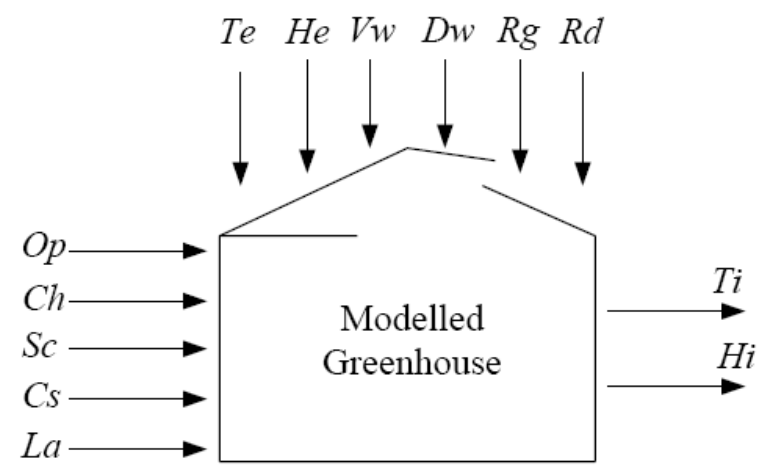

Figure 3: Block diagram of the greenhouse

\subsection{DATA SETS}

Concerning the used data sets, the sampling time for measures acquisition has been 1 minute, which corresponds to 1440 measurement points during 24 hours.

Data corresponding to situations like deactivation of some actuator, failures of power supply, lack of culture or malfunctions of the control system, are not useful for the purpose of the present work, so have been discarded. Using data from different seasons provides relevant information about effects and interactions of the actuators and outside climate conditions. Besides, the control policy, defined by user setpoints, is modified depending on the season and, mainly, on the nature of the culture. This way, poor extrapolation properties associated to new weather conditions and/or new control actions pointed by Linker et al. [12], are avoided.

The utilized database contains a monthly set of 7 days parameters values for two months of the summery season (August and May) and for two months of the wintry season (November and February).

Red pepper plants of cultivar Jaranda were grown in the greenhouse whereas all experiment data were collected. Therefore, greenhouse environmental behavior was influenced by the changes and evolution of plant physiological processes, making the models under development to be adapted to real conditions of greenhouse utilization.

Involved variables have different nature (analogue signals and Boolean commands), ranges and dimensions, so input and output data are scaled to an interval of amplitude two $[-1,+1]$. This pretreatment prevents from losing the contribution of small-range variables and tends to make the training process better behaved [15].

\section{DEVELOPED NEURAL NETWORK-BASED MODELS}

\subsection{NEURAL NETWORKS}

The complex and nonlinear nature presented by greenhouses, makes hard to build models that represent the whole dynamics of the system. For this reason, advanced techniques for nonlinear dynamic systems are used to solve such problem. In our case, neural networks have been chosen to deal with greenhouse modeling, since they have demonstrated in previous studies to be useful and powerful tools to identify such system.

The identification process by means of neural networks is called "black box" modeling, based on measured data, without need of knowledge of internal functioning of the system to be modeled.

Neural network technology is a form of artificial intelligence that "learns" by processing representative data patterns through its internal architecture. It is based on theories of the massive interconnection and parallel processing architecture of biological neural systems.

The use of neural networks requires several important choices made in the search of a model that provides satisfactory results. The main choices are the topology, the algorithm of learning, the number of hidden neurons, and the selection of input variables set to be used. In general, these design questions have no straightforward answer, and there is no systematic method to find an optimal structure of a neural network.

Temperature and humidity play a key role in greenhouse climate so they constitute the network outputs for every model under study. This way, the models will try to predict the inside air temperature and relative humidity in the greenhouse as a function of the variables used as input for the network, as outside air temperature, relative humidity, solar radiation, etc., and the actuators state signals, as window opening, screen position, heating operation, and others.

To compare these models, several criteria are used, considering the prediction error, such as the Mean Squared Error (MSE), and some factors related with the network architecture, such as the number of input variables, the number of hidden neurons and others.

\subsection{RESULTS}

The developed models are Multilayered Perceptron (MLP) neural networks (Figure 4), which is the most commonly used neural network architecture. 
Namely, as it has been commented in the previous subsection, two neural network-based models have been developed to predict the inside temperature and relative humidity, respectively.

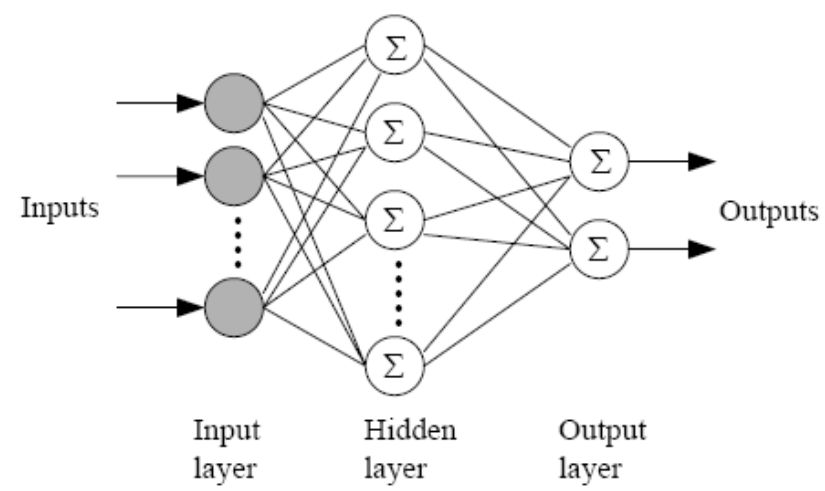

Figure 4: Block diagram of the MLP neural network

Both models are composed by three layers, consisting of an input layer, one hidden layer with 10 neurons, and an output layer with 2 nodes. Input variables in this case are the following: external temperature and relative humidity, solar radiation, window opening, screen position, heating state and lighting lamps state. The used algorithm to adjust the weight and bias values is the Levenberg-Marquardt algorithm. The transfer function of the hidden layer is of sigmoid type, and the output layer has a linear transfer function. Available data patterns have been divided so that $60 \%$ of the samples are used for training, 20\% for validation and $20 \%$ for testing.

The different models have been tested and implemented under the environment Matlab, using the Neural Network Toolbox.

Figure 5 and Figure 6 show the comparison curves between measured and predicted values of the inside temperature and relative humidity for two days on February.

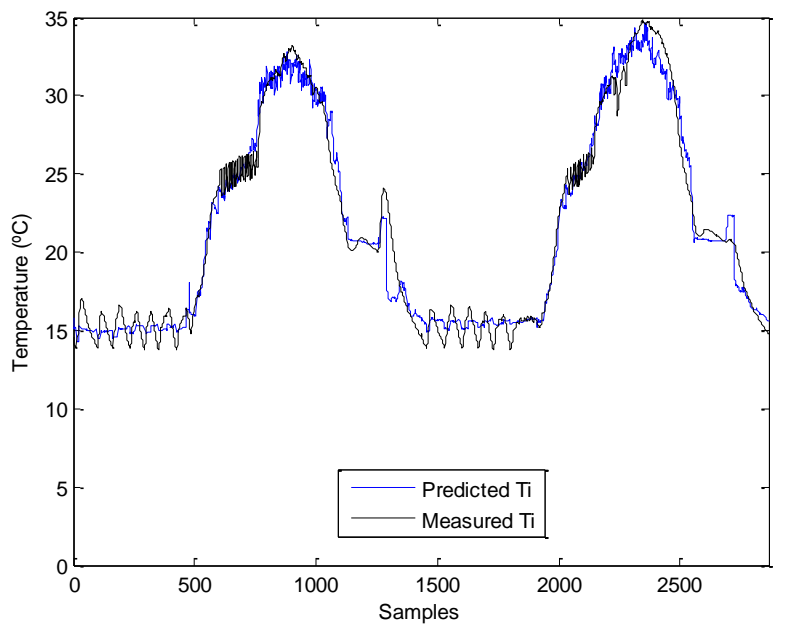

Figure 5: Measured and predicted inside temperature through the MLP neural network

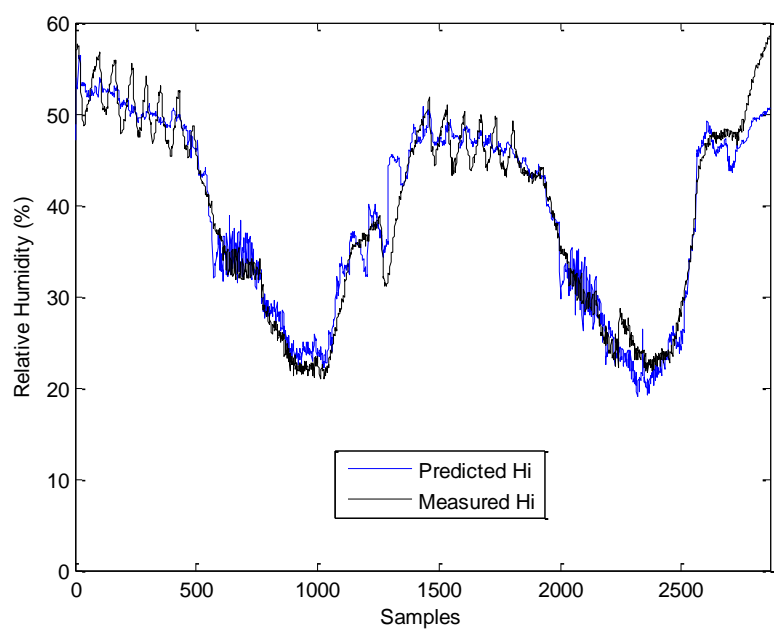

Figure 6: Measured and predicted inside relative humidity through the MLP neural network

As can be seen in both figures, the evolution of the measured and predicted magnitudes is satisfactory. The corresponding MSE are $1.12^{\circ} \mathrm{C}$ for temperature and $6.57 \%$ for relative humidity, indicating that predictions obtained from the neural models present a good behavior; therefore, both neural network-based models have been successfully validated.

\section{CONCLUSIONS}

This paper has presented the design and validation of two models based on neural networks to predict the inside temperature and relative humidity of a greenhouse. The presented results prove a good performance with low MSE values. Although, it is necessary to evaluate and compare different configurations of such models in order to improve their performance and generalization capability.

Moreover, these models are envisioned to be used as heart for model-based control strategies implemented by a supervisory control system with the goal of improving the greenhouse behaviour.

\section{Acknowledgments}

This work has been supported by the FEDER Funds (Programa Operativo FEDER de Extremadura 20142020) through the grant "Ayuda a Grupos de Investigación" (ref. GR18159) of Junta de Extremadura. 


\section{References}

[1] Bennis, N., Duplaix, J., Enéa, G., Haloua, M., Youlal, H., (2008) "Greenhouse Climate Modelling and Robust Control", Computers and Electronics in Agriculture, vol. 61, pp. 96-107.

[2] Boaventura Cunha, J., "Greenhouse Climate Models: an Overview", Proceedings of the EFITA 2003 Conference, pp. 823-829, 2003.

[3] Bot, G.P.A., (1991) "Physical Modelling of Greenhouse Climate", Proceedings of the IFAC/ISHS Workshop, pp. 7-12.

[4] Boulard, T., Baille, A., (1993) "A simple Greenhouse Climate Control Model incorporating effects on Ventilation and Evaporative Cooling", Agricultural and Forest Meteorology, 65, pp. 145-157.

[5] Castañeda-Miranda, A., Castaño, V.M., (2017) "Smart frost control in greenhouses by neural networks models, Computers and Electronics in Agriculture, vol. 137, pp. 102-114.

[6] Dariouchy, A., Aassif, E., Lekouch, K., Bouirden, L., Maze, G., (2009) "Prediction of the Intern Parameters Tomato Greenhouse in a Semi-arid Area using a Time-series Model of Artificial Neural Networks", Measurement, vol. 42, pp. 456-463.

[7] Ferreira, P.M., Faria, E.A., Ruano, A.E., (2002) "Neural Network Models in Greenhouse Air Temperature Prediction", Neurocomputing, vol. 43, pp. 51-75.

[8] Ferreira, P.M., Ruano, A.E., Fonseca, C.M., (2003) "Genetic Assisted Selection of RBF Model Structures for Greenhouse Inside Air Temperature Prediction", Proceedings of the 2003 IEEE Conference on Control Applications, pp. 576-581.

[9] Fourati, F., Chtourou, M., (2007) "A Greenhouse Control with Feed-Forward and Recurrent Neural Networks", Simulation Modelling Practice and Theory, vol. 15, pp. 1016-1028.

[10] He, F., Ma, C., Zhang, J., Chen, Y., (2007) "Greenhouse Air Temperature and Humidity Prediction Based on Improved BP Neural Network and Genetic Algorithm", Proceedings of the 4th International Symposium on Neural Networks, LNCS vol. 4493, pp. 973-980.

[11] Linker, R., Seginer, I., (2004) “Greenhouse temperature modeling: a comparison between sigmoid neural networks and hybrid models", Mathematics and Computers in Simulation, vol. 65 , pp. 19-29.

[12] Linker, R., Seginer, I., Gutman, P.O., (1998) "Optimal CO2 Control in a Greenhouse Modeled with Neural Networks", Computers and Electronics in Agriculture, vol. 19, pp. 289-310.

[13] Rodríguez, F., (2002) "Modelado y Control Jerárquico de Crecimiento de Cultivos en Invernadero", $\mathrm{PhD}$ Thesis, University of Almería.

[14] Salgado, P., Boaventura Cunha, J., (2005) "Greenhouse Climate Hierarchical Fuzzy Modelling", Control Engineering Practice, Vol. 13, pp. 613-628.

[15] Sarle, W.S., (2002) "Neural Frequently Asked Questions".

http://ftp.sas.com/pub/neural/FAQ.html.

[16] Taki, M., Ajabshirchi, Y., Faramarz Ranjbar, S., Rohani, A., Matloobi, M. (2016) "Heat transfer and MLP neural network models to predict insideenvironment variables and energy lost in a semi-solar greenhouse", Energy and Buildings, vol. 110, pp. 314-329.

[17] Uchida, H., Pieters, J.G., (2004) "Modelling Greenhouse Temperature using System Identification by means of Neural Networks", Neurocomputing, vol. 56, pp. 423-428.

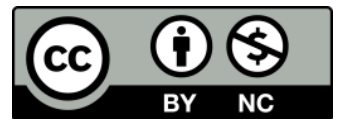

(C) 2018 by the authors. Submitted for possible open access publication under the terms and conditions of the Creative Commons Attribution CC-BY-NC 3.0 license (https://creativecommons.org/licenses/by-nc/3.0). 